\title{
Correlation between interleukin-6, interleukin-8, and modified early warning score of patients with acute ischemic stroke and their condition and prognosis
}

\author{
Liya Zhang, Dongxun Xu, Tianzhao Zhang, Wei Hou, Layong Yixi \\ Department of Neurology, 363 Hospital, Chengdu, China \\ Contributions: (I) Conception and design: L Zhang; (II) Administrative support: D Xu; (III) Provision of study materials or patients: L Zhang, D Xu, \\ T Zhang, L Yixi; (IV) Collection and assembly of data: All authors; (V) Data analysis and interpretation: L Zhang, T Zhang, W Hou, L Yixi; (VI) \\ Manuscript writing: All authors; (VII) Final approval of manuscript: All authors. \\ Correspondence to: Liya Zhang. Department of Neurology, 363 Hospital, Daosangshu Road 108\#, Chengdu 610041, China. Email: yalizhang1@sina.com.
}

Background Acute ischemic stroke (AIS) is closely related to the level of inflammatory factors. This study aimed to explore the correlation between interleukin-6 (IL-6), interleukin-8 (IL-8), and the modified early warning score (MEWS) of AIS patients and their condition and prognosis.

Methods: The clinical data of 95 AIS patients admitted to our hospital from January 2019 to October 2019 were selected, and 91 cases were finally recruited to the study group according to the inclusion and exclusion criteria. A control group was recruited comprising 70 healthy patients. The differences in IL-6 and IL-8 levels between the 2 groups were compared. Multiple logistic regression analysis was used to analyze the independent risk factors affecting the prognosis of AIS patients. A receiver-operating characteristic (ROC) curve was used to analyze the predictive value of IL-6, IL-8, and MEWS for the poor prognosis of AIS patients.

Results: The levels of IL-6 and IL-8 in the study group were higher than those of the control group $(\mathrm{P}<0.05)$. After 90 days of treatment, 69 cases in the study group allocated into the good prognosis group, and 22 were allocated into the poor prognosis group. The National Institutes of Health Stroke Scale (NIHSS) scores before thrombolysis, blood glucose before thrombolysis, systolic blood pressure $2 \mathrm{~h}$ after thrombolysis, IL-6, IL-8, and MEWS scores within $24 \mathrm{~h}$ of admission in the good prognosis group were lower than those of the poor prognosis group $(\mathrm{P}<0.05)$. The area under the curve (AUC) of IL-6, IL-8, MEWS, and the 3 combined curves were $0.937,0.897,0.839$, and 0.976 , respectively, and the area under the combined detection curve was the largest.

Conclusions: The inflammatory response and secondary brain damage after AIS are influenced by IL-6 and IL-8. Combined with the MEWS score, IL-6 and IL-8 can be used as important indicators to judge the severity of the early condition of AIS patients. The combination of these 3 indicators has high accuracy in evaluating the prognosis of patients and is worthy of clinical promotion.

Keywords: Acute ischemic stroke (AIS); interleukin-6 (IL-6); interleukin-8 (IL-8); improved early warning score; prognosis; correlation analysis.

Submitted Sep 18, 2020. Accepted for publication Nov 26, 2020.

doi: 10.21037/apm-20-2200

View this article at: http://dx.doi.org/10.21037/apm-20-2200 


\section{Introduction}

Acute ischemic stroke (AIS) is a series of clinical syndromes of neurological dysfunction, in which the blood supply of brain tissue is impaired due to various reasons, resulting in ischemic hypoxic necrosis (1). The incidence of AIS is high, and it progresses rapidly; accurate evaluation of the patient's condition at the early stage is critical for effective intervention in the disease progression (2). The modified early warning score (MEWS) is a physiological scoring system for early identification of critically ill patients or potentially critically ill patients, and has been widely used in clinical practice (3).

Besides, the pathogenesis of AIS is predominantly related to atherosclerosis. Atherosclerosis itself and necrotic brain tissue can activate inflammatory cells to release inflammatory mediators and aggravate tissue damage, which means the level of inflammatory factors is closely related to the condition and prognosis of AIS patients (3). Interleukin-6 (IL-6) and interleukin-8 (IL-8) are markers of the acute inflammatory response, and therapy both promoting and diminishing interleukin levels is becoming increasingly applied clinically (4). However, although the value of IL-6 and IL-8 in diagnosis, treatment, and prognostic evaluation is significant, the correlation between ASI and the three (IL-6, IL-8, MEWS) has not been reported in China. Therefore, this study analyzed the correlation between IL-6, IL-8, and MEWS in patients with AIS and their condition and prognosis, in order to gather ideas for clinical treatment. We present the following article in accordance with the STARD reporting checklist (available at http:// dx.doi.org/10.21037/apm-20-2200).

\section{Methods}

\section{General information}

The clinical data of 95 AIS patients admitted to our hospital from January 2019 to October 2019 were selected as study participants. The inclusion criteria were: (I) all were diagnosed by magnetic resonance imaging (MRI) and other examinations to meet the AIS diagnostic criteria; (II) participants were at their first onset of AIS, with no history of cerebral infarction or cerebral hemorrhage; (III) complete clinical data available; (IV) participants aged $18-80$ years old. Participants were excluded if any of the following: (I) comorbidity of other hemorrhagic brain diseases or other brain diseases, such as aneurysm, tumor, or intracranial infection; (II) had been taking anticoagulant drugs before admission; (III) a history of thrombolysis; (IV) incomplete clinical data; (V) insufficiency of vital organs such as liver and kidney or other infectious diseases. Finally, a total of 91 cases who met all the inclusion and exclusion criteria of this study constituted the study group, which included 40 males and 51 females, aged $35-70$ years (average age $61.75 \pm 8.96$ years old).

Concurrently, 70 patients with normal health examination results and complete clinical data in the same hospital during the same period were included into the control group, which included 36 males and 34 females, aged $32-72$ years (average age $61.39 \pm 8.71$ years). There was no significant difference in general information such as gender or age between the two groups $(\mathrm{P}>0.05)$. All procedures performed in this study involving human participants were in accordance with the Declaration of Helsinki (as revised in 2013). This study was approved by the 363 Hospital (No. 2020-30). Informed consent was taken from all the patients.

\section{Research methods}

Clinical data of all participants were collected, and the differences in IL- 6 and IL- 8 levels between the 2 groups were compared. The prognosis of patients in the study group were recorded, and then multivariate logistic regression analysis was used to analyze the independent risk factors affecting the prognosis of AIS patients. And finally, a receiver-operating characteristic (ROC) curve was used to analyze the predictive value of IL- 6 , IL-8, and MEWS for the poor prognosis of AIS patients.

\section{Observation indicators}

\section{Detection of IL-6 and IL-8 levels}

The cubital venous blood of patients in the study group was collected on admission, and fasting venous blood of $3 \mathrm{~mL}$ was collected in the control group in the morning. After standing for $30 \mathrm{~min}$ at room temperature, the blood samples were centrifuged $(1,500 \mathrm{r} / \mathrm{min}, 20 \mathrm{~min})$ to separate the supernatant. Then the level of serum IL- 6 and IL- 8 were detected using double antibody sandwich enzyme-linked immunosorbent assay (ELISA) with detection kits (YES biotech laboratory Ltd., Mississauga, ON, Canada), and the results were measured with AU5800 automatic biochemical analyzer (Beckman Coulter Inc., Brea, CA, USA).

\section{MEWS score (5)}

After patient recruitment, a MEWS score sheet was used 
Table 1 Comparison of IL-6 and IL-8 levels in the two groups $\left(\bar{x}_{ \pm} \mathrm{SD}, \mathrm{pg} / \mathrm{mL}\right)$

\begin{tabular}{lcc}
\hline Group & $\mathrm{IL}-6$ & $\mathrm{IL}-8$ \\
\hline Study group $(\mathrm{n}=91)$ & $1.46 \pm 0.51$ & $4.95 \pm 0.46$ \\
Control group $(\mathrm{n}=70)$ & $0.68 \pm 0.16$ & $2.57 \pm 0.25$ \\
$t$ & 12.330 & 35.060 \\
$\mathrm{P}$ & $<0.001$ & $<0.001$ \\
\hline
\end{tabular}

IL-6, interleukin-6; IL-8, interleukin-8; $\bar{x} \pm \mathrm{SD}$, mean \pm standard deviation.

by the same nursing staff to obtain the MEWS score according to the clinical data of participants in the study group, including mental response, systolic blood pressure, heart rhythm, respiratory rate, and body temperature. The scores of each physiological indicator were $0-3$ points, and the total scores were 15 points. The higher the score, the more serious the potential condition of the patient. When MEWS $\geq 8$ points, it indicated that the patient is at very high risk.

\section{Prognosis evaluation}

After admission, all patients underwent imaging examinations such as MRI. At the same time, active medical treatments were performed, including blood pressure maintenance, diuretics to reduce intracranial pressure and cerebral edema, vasodilators to dilate blood vessels, anticoagulant therapy, calcium antagonists and intravenous thrombolytic therapy. The prognosis of thrombolysis was evaluated by the 90-day modified Rankin Scale (mRS) scores after the onset of ASI (6): patients with $0-1$ point indicated a good prognosis of thrombolysis and were allocated to the good prognosis group, while patients with 2-6 points indicated poor prognosis of thrombolysis and were assigned to the poor prognosis group.

\section{Statistical methods}

The data in this study were analyzed using SPSS version 18.0 software (IBM Corp., Armonk, NY, USA). The count data were expressed as $\mathrm{n}(\%)$, and analyzed using $\mathrm{F} / \mathrm{t}$-test, and the measurement data were described as mean \pm standard deviation $\left(\bar{x}_{ \pm} \mathrm{SD}\right)$, and analyzed by chi-squared $\left(\chi^{2}\right)$ test. Multiple logistic regression was performed to analyze the independent risk factors affecting the prognosis of AIS patients. The ROC curve was used to analyze the predictive value of IL-6, IL-8, and MEWS for poor prognosis of AIS patients. Results with $\mathrm{P}<0.05$ were considered to be statistically significant.

\section{Results}

\section{Comparison of IL-6 and IL-8 levels between groups}

The levels of IL-6 and IL-8 in the study group were higher than those in the control group $(\mathrm{P}<0.05$, Table 1).

\section{Prognosis of AIS patients (91 cases)}

After 90 days of treatment, a total of 69 cases among the 91 patients in the study group had a good prognosis, and 22 cases had a poor prognosis, with a poor prognosis rate of $27.16 \%$. According to the prognosis status, the participants were allocated into the good prognosis group $(n=69)$ and the poor prognosis group $(n=22)$.

\section{Single factor analysis of the prognosis of AIS patients}

There was no significant difference between the good prognosis group and poor prognosis group in gender, body mass index (BMI), history of hypertension, and diastolic pressure $2 \mathrm{~h}$ after thrombolysis $(\mathrm{P}>0.05)$. however, there were more patients aged $<60$ years old and with no history of diabetes and smoking in the good prognosis group than those in the poor prognosis group. The National Institutes of Health Stroke Scale (NIHSS) scores and blood glucose before thrombolysis, systolic pressure $2 \mathrm{~h}$ after thrombolysis, IL-6 and IL-8 levels, and MEWS scores within $24 \mathrm{~h}$ of admission in the good prognosis group were lower than those in the poor prognosis group $(\mathrm{P}<0.05$, Table 2).

\section{Analysis of multiple factors affecting the prognosis of AIS patients}

NIHSS score before thrombolysis, systolic pressure $2 \mathrm{~h}$ after thrombolysis, IL-6, IL-8, and MEWS scores within $24 \mathrm{~h}$ of admission were all independent risk factors that affected the prognosis of AIS patients $(\mathrm{P}<0.05$, Table 3).

\section{Analysis of the predictive value of IL-6, IL-8 levels, and MEWS score on the poor prognosis of AIS patients}

The ROC curve was used to analyze the predictive value of 
Table 2 Single factor analysis of the prognosis of AIS patients

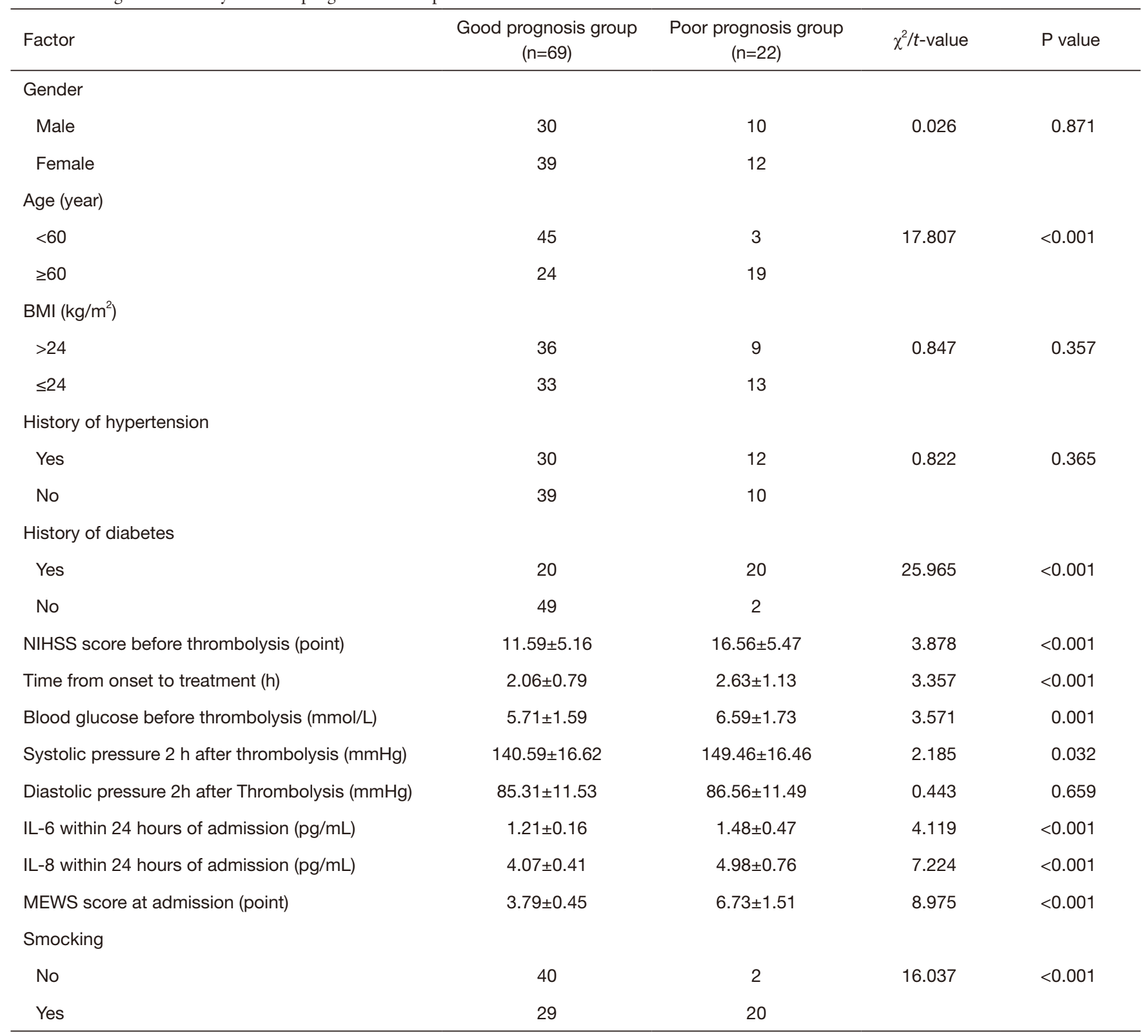

AIS, acute ischemic stroke; IL-6, interleukin-6; IL-8, interleukin-8; NIHSS, National Institutes of Health Stroke Scale; BMI, body mass index; MEWS, modified early warning score.

IL-6 and IL-8 levels, MEWS score, and the combination of the 3 indicators on the poor prognosis of patients with AIS. The area under the curve (AUC) of IL-6, IL-8, MEWS, and the combination of the 3 were $0.937,0.897,0.839$, and 0.976, respectively, and the AUC of the combination of the 3 indicators was the largest (Table 4 and Figure 1).

\section{Discussion}

In clinical practice, AIS is the most common cardiovascular disease. Patients with AIS often show clinical symptoms such as hemiplegia and aphasia, which seriously reduces their quality of life, and threatens their health (7). In recent years, with cumulative annual increases in the prevalence, 
Table 3 Analysis of multiple factors affecting the prognosis of AIS patients

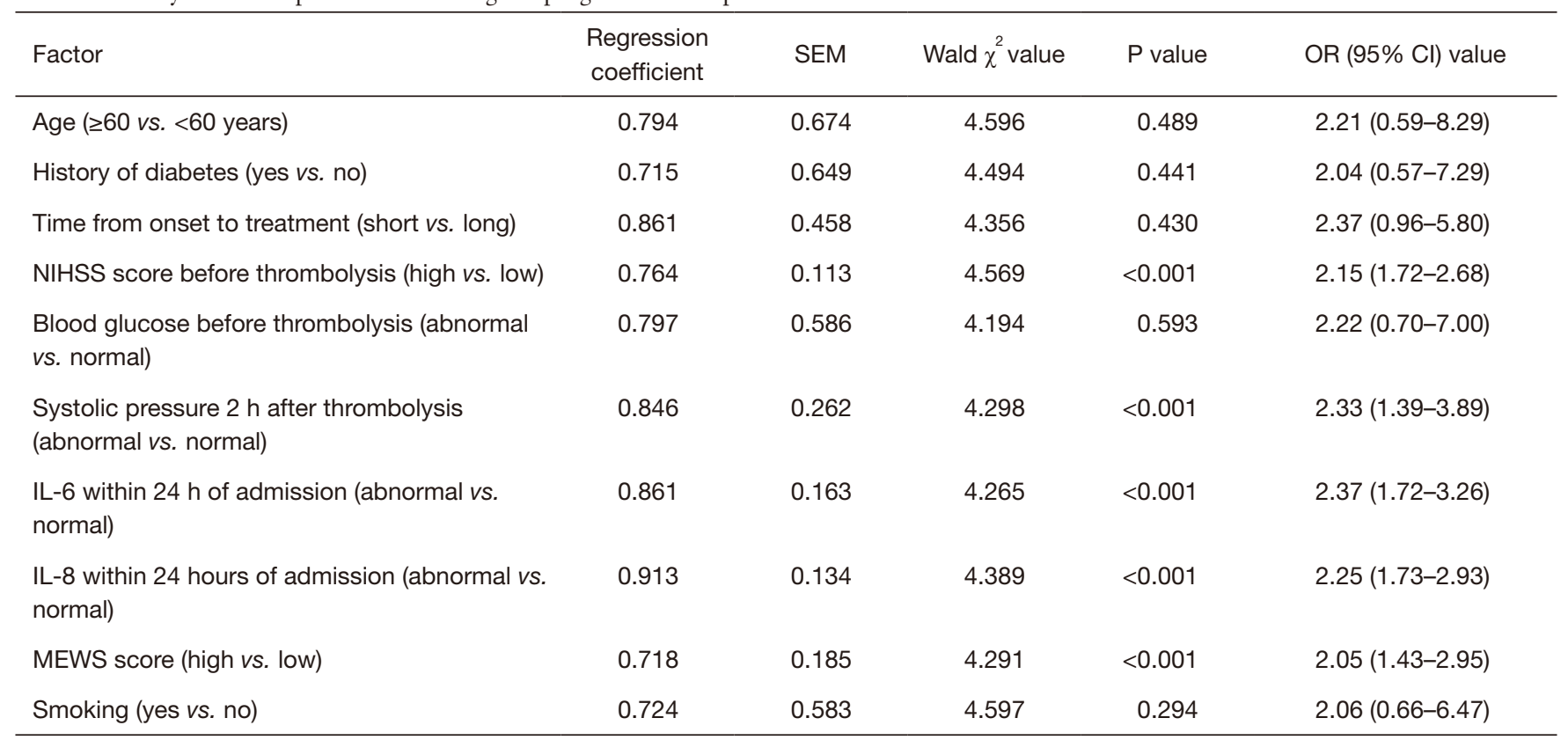

SEM, standard error of mean; Cl, confidence interval; IL-6, interleukin-6, IL-8, interleukin-8; NIHSS, National Institutes of Health Stroke Scale; MEWS, modified early warning score; OR, odds ratio.

Table 4 Analysis of the predictive value of IL-6, IL-8, MEWS, and the combined detection of the 3 indicators on the poor prognosis of AIS patients

\begin{tabular}{lccccc}
\hline Predicted indicator & Optimal threshold & Sensitivity & Specificity & AUC of ROC & $95 \%$ Cl \\
\hline IL-6 & 26.56 & 0.886 & 0.865 & 0.937 & $0.846-1.000$ \\
IL-8 & 37.61 & 0.856 & 0.856 & 0.897 & $0.783-0.975$ \\
MEWS & 37.46 & 0.836 & 0.837 & 0.839 & $0.718-0.960$ \\
Combination of the three & 38.15 & 0.926 & 0.964 & 0.976 & 0.879 \\
Predictive exponential model & 1.89 & 0.895 & 0.769 & $0.763-0.869$ \\
\hline
\end{tabular}

AUC, area under the curve; ROC, receiver-operating characteristic; IL-6, interleukin-6; IL-8, interleukin-8; MEWS, modified early warning score; $\mathrm{Cl}$, confidence interval.

disability, and mortality rates of AIS, strategies to improve the therapeutic effect and ameliorate the poor prognosis of AIS patients have highly sought after (8).

Accurate evaluation of the condition of AIS patients before hospitalization is essential to ensure that patients receive effective treatment. At present, means of assessing the severity of clinically critically ill patients mostly relies on the Acute Physiology and Chronic Health Score II, Simplified Acute Physiological State Score System, the Glasgow Coma Score, and some other score systems (9). Although these indicators can predict the prognosis of critically ill patients to a certain extent, due to the large volume of evaluation data and the multitude of impacting factors on the scores such as environment, time, and human resources, they are not suitable to evaluate the condition of critically ill patients in pre-hospital emergency situations. The MEWS is an improved scoring system based on the British Early Warning Score developed by the Royal College of Physicians, which is mainly used to predict the trend of critical illness in hospitals at all levels (10). The MEWS score is attained using the 5 vital signs of the patient as evaluation indicators, including systolic pressure, heart rhythm, respiratory rate, body temperature, and consciousness, which provides the advantages of accuracy, 


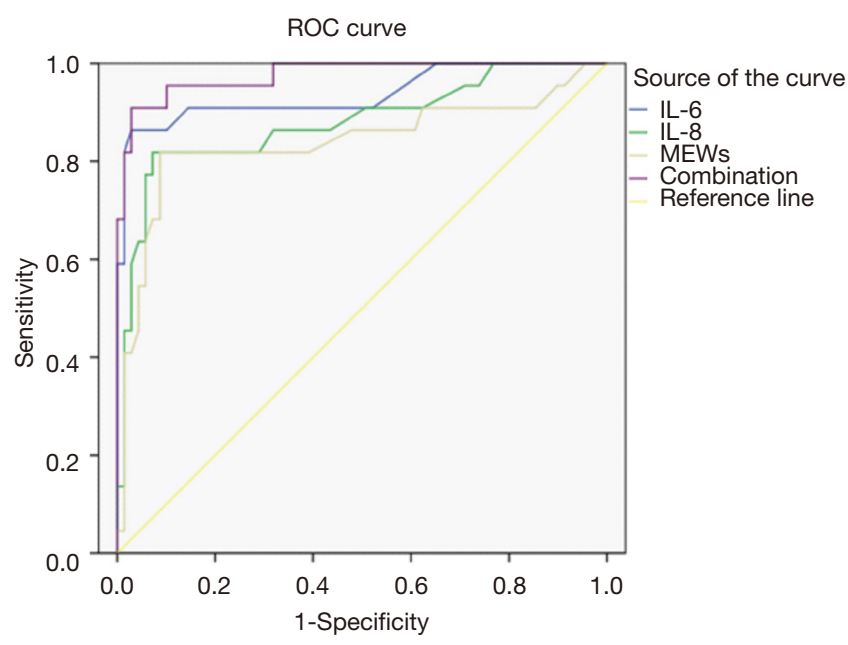

Figure 1 Analysis of the predictive value of IL-6, IL-8, and MEWS for poor prognosis of AIS patients. IL-6, interleukin-6; IL-8, interleukin-8; MEWS, modified early warning score; AIS, acute ischemic stroke.

speed, and practicality. The most prominent application of MEWS is evaluation of the early onset of AIS that the patients can complete within a few minutes of hospital admission. The evaluation has high application value because it is not affected by the emergency environment, manpower, and other factors, and has high repetitive operability (11). Hydes et al. have reported that MEWS score more accurately evaluates the condition of patients with cardiac arrest, and may have a certain relationship with the prognosis of patients (12). The results of this study showed that the MEWS score was significantly increased for AIS patients in the poor prognosis group, and was an independent risk factor affecting the prognosis of AIS patients $(\mathrm{P}<0.05)$. These findings supported the work of Hydes et al. by indicating that the MEWS score could evaluate the condition of AIS patients accurately, and that it may have a certain relationship with patient prognosis.

At present, it is believed that the pathogenesis of AIS includes energy expenditure, nitric oxide and free radical damage, along with inflammatory cytokine hypotheses. With the proposal of the injury-response theory, increasing numbers of studies have begun to focus on the correlation between AIS and inflammation (13). Atherosclerosis is the main pathogenic factor of AIS that can cause vascular obstruction, leading to cerebral ischemia and hypoxia. Atherosclerotic plaque and necrotic brain tissue can activate inflammatory cells and induce auto-inflammatory responses. Therefore, local and systemic inflammatory responses are closely related to atherosclerosis (14).

As a pleiotropic cytokine, IL-6 could be mainly produced by $\mathrm{T}$ cells, B cells, monocytes, and endothelial cells, as well as astrocytes and microglia in brain tissue (15). Under normal physiological conditions, IL-6 and its receptors are continuously expressed at low concentrations in brain tissues, participating in various physiological activities such as mediating the central immune system, nerve repair, coordinated metabolism, and neuroendocrine. When cerebral ischemia occurs, IL-6 can be activated to become continuously highly expressed. On the one hand, it performs physiological functions such as neuroprotection. On the other hand, as an important inflammatory mediator, it may participate in secondary brain injury (16). A chemotactic cytokine, IL-8 is mainly produced by activated monocytes, macrophages, and endothelial cells. It acts as a potential polymorphonuclear leukocyte chemotactic to induce neutrophils to accumulate at ischemic sites, thereby causing inflammation and edema both in vivo and in vitro $(17,18)$. In addition, interleukin can significantly stimulate and improve the activity of acetylcholinesterase in characteristic brain regions, reduce the survival of acetylcholine, and thus affect the cognitive functions of patients, which is not conducive to the recovery of prognosis. The results of this study showed that the levels of IL-6 and IL-8 in AIS patients were higher than those in the normal healthy group at each interval, and the levels of IL- 6 and IL- 8 in the study group reached their peak at $24 \mathrm{~h}$ after admission, and then gradually decreased $(\mathrm{P}<0.05)$. Results of multivariate analysis also indicated that IL-6 and IL-8 were independent risk factors affecting the prognosis of AIS patients $(\mathrm{P}<0.05)$, and were closely related to the prognosis of AIS patients; therefore, it was suggested that serum IL-6 and IL- 8 levels can be used as important indicators to assess the disease progression of AIS at the early stage, and have a certain value in predicting prognosis.

The ROC curve was further used to analyze the predictive value of IL-6, IL-8, and MEWS on poor prognosis of AIS patients. The results showed that the AUC of the combination of the 3 indicators was the largest among the multiple indicators, signaling that the combination of the 3 indicators had the highest predictive value. Therefore, the combined detection of the 3 indicators is an effective way to predict the poor prognosis of AIS patients.

\section{Conclusions}

In summary, IL-6 and IL-8 are involved in inflammation 
and secondary brain damage that follow the onset of AIS. The combination of IL-6, IL-8, and MEWS score can be used as an important indicator for judging the severity of AIS at the early stage, it showed high accuracy in evaluating the prognosis of AIS patients, and is worthy of clinical adoption.

\section{Acknowledgments}

Funding: None.

\section{Footnote}

Reporting Checklist: The authors have completed the STARD reporting checklist. Available at http://dx.doi.org/10.21037/ apm-20-2200

Data Sharing Statement: Available at http://dx.doi. org/10.21037/apm-20-2200

Conflicts of Interest: All authors have completed the ICMJE uniform disclosure form (available at http://dx.doi. org/10.21037/apm-20-2200). The authors have no conflicts of interest to declare.

Ethical Statement: The authors are accountable for all aspects of this work in ensuring that questions related to the accuracy or integrity of any part of the work are appropriately investigated and resolved. This study was approved by the 363 Hospital (No. 2020-30). All procedures performed in this study involving human participants were in accordance with the Declaration of Helsinki (as revised in 2013). Informed consent was taken from all the patients.

Open Access Statement: This is an Open Access article distributed in accordance with the Creative Commons Attribution-NonCommercial-NoDerivs 4.0 International License (CC BY-NC-ND 4.0), which permits the noncommercial replication and distribution of the article with the strict proviso that no changes or edits are made and the original work is properly cited (including links to both the formal publication through the relevant DOI and the license). See: https://creativecommons.org/licenses/by-nc-nd/4.0/.

\section{References}

1. Saab A, Glass-Kaastra S, Young GB. Discharge Destination from a Rehabilitation Unit After Acute Ischemic Stroke. Can J Neurol Sci 2019;46:209-15.

2. Kowalczyk K, Jabłoński B, Kwarciany M, et al. Changes of augmentation index early after ischaemic stroke predict functional outcome. Blood Press 2020;29:327-35.

3. Song K, Guan M, Li W, et al. Acute ischemic stroke patients with diffusion-weighted imaging-Alberta Stroke Program Early Computed Tomography Score $\leq 5$ can benefit from endovascular treatment: a singlecenter experience and literature review. Neuroradiology 2019;61:451-9.

4. Tan C, Liu X, Mo L, et al. Statin, cholesterol, and sICH after acute ischemic stroke: systematic review and metaanalysis. Neurol Sci 2019;40:2267-75.

5. Huang ZX, Wang QZ, Dai YY, et al. Early neurological deterioration in acute ischemic stroke: A propensity score analysis. J Chin Med Assoc 2018;81:865-70.

6. Day AL, Branch CL, Giannotta SL. Commentary: Modern Training and Credentialing in Neuroendovascular Acute Ischemic Stroke Therapy. Neurosurgery 2019;85:S58-9.

7. Zhu L, Gong S, Zhu X, et al. FLAIR vascular hyperintensity: an unfavorable marker of early neurological deterioration and short-term prognosis in acute ischemic stroke patients. Ann Palliat Med 2020;9:3144-51.

8. Foley C, Dowling M. How do nurses use the early warning score in their practice? A case study from an acute medical unit. J Clin Nurs 2019;28:1183-92.

9. Smith CJ, Hulme S, Vail A, et al. SCIL-STROKE (Subcutaneous Interleukin-1 Receptor Antagonist in Ischemic Stroke): A Randomized Controlled Phase 2 Trial. Stroke 2018;49:1210-6.

10. Dieplinger B, Bocksrucker C, Egger M, et al. Prognostic Value of Inflammatory and Cardiovascular Biomarkers for Prediction of 90-Day All-Cause Mortality after Acute Ischemic Stroke-Results from the Linz Stroke Unit Study. Clin Chem 2017;63:1101-9.

11. Romero-Ortuno R, Wallis S, Biram R, et al. Clinical frailty adds to acute illness severity in predicting mortality in hospitalized older adults: An observational study. Eur J Intern Med 2016;35:24-34.

12. Hydes TJ, Meredith P, Schmidt PE, et al. National Early Warning Score Accurately Discriminates the Risk of Serious Adverse Events in Patients with Liver Disease. Clin Gastroenterol Hepatol 2018;16:1657-1666.e10.

13. Prakash N, Stumbles P, Mansfield CS. Concentrations of interleukin- $6,-8,-10$ and tumour necrosis factor- $\alpha$ in the faeces of dogs with acute diarrhoea. N Z Vet J 2019;67:138-42. 
14. Voiriot G, Razazi K, Amsellem V, et al. Interleukin-6 displays lung anti-inflammatory properties and exerts protective hemodynamic effects in a double-hit murine acute lung injury. Respir Res 2017;18:64.

15. Tiedt S, Prestel M, Malik R, et al. RNA-Seq Identifies Circulating miR-125a-5p, miR-125b-5p, and miR-143-3p as Potential Biomarkers for Acute Ischemic Stroke. Circ Res 2017;121:970-80.

16. Chen X, Wang Y, Fu M, et al. Plasma Immunoproteasome Predicts Early Hemorrhagic Transformation in Acute Ischemic Stroke Patients. J Stroke Cerebrovasc Dis 2017;26:49-56.

Cite this article as: Zhang L, Xu D, Zhang T, Hou W, Yixi L. Correlation between interleukin-6, interleukin-8, and modified early warning score of patients with acute ischemic stroke and their condition and prognosis. Ann Palliat Med 2021;10(1):148155. doi: 10.21037/apm-20-2200
17. Kozak HH, Uğuz F, Kılınç İ, et al. Delirium in patients with acute ischemic stroke admitted to the non-intensive stroke unit: Incidence and association between clinical features and inflammatory markers. Neurol Neurochir Pol 2017;51:38-44.

18. Huttunen K, Siponen T, Salonen I, et al. Low-level exposure to ambient particulate matter is associated with systemic inflammation in ischemic heart disease patients. Environ Res 2012;116:44-51.

(English Language Editor: J. Jones) 